\title{
Mechanism of Baihe Decoction in the treatment of coronary heart disease based on network pharmacology and molecular docking
}

\author{
Yaxian Jin ${ }^{1,2}$, Xiaodan Yin $^{1}$, Zhenying $\mathrm{Li}^{1}, \mathrm{Jiangyan} \mathrm{Xu}^{1}$ \\ ${ }^{1}$ Henan University of Chinese Medicine, Zhengzhou, China; ${ }^{2}$ First Affiliated Hospital of Guizhou University of Traditional Chinese Medicine, \\ Guiyang, China \\ Contributions: (I) Conception and design: Y Jin; (II) Administrative support: J Xu; (III) Provision of study materials or patients: X Yin; (IV) Collection \\ and assembly of data: Y Jin, X Yin, Z Li; (V) Data analysis and interpretation: Y Jin, J Xu; (VI) Manuscript writing: All authors; (VII) Final approval \\ of manuscript: All authors. \\ Correspondence to: Jiangyan Xu. Henan University of Chinese Medicine, 156 Jinshui East Road, Zhengdong New District, Zhengzhou 450046, China. \\ Email: jinyaxian2020@163.com.
}

Background: Network pharmacology is widely used in mechanistic studies of traditional Chinese medicines (TCMs). The present study aimed to predict the target and signaling pathway of Baihe Decoction in the intervention of coronary heart disease (CHD) based on a network pharmacology approach and molecular docking.

Methods: The active ingredients of Baihe Decoction were screened by the Traditional Chinese Medicine Systems Pharmacology (TCMSP), and their potential target genes and proteins in CHD were predicted. The targets were screened out using Online Mendelian Inheritance in Man and the Genecards database. Venn soft was used to obtain the common targets of drugs and diseases. The compound-target-disease network of Baihe Decoction in CHD was constructed in Cytoscape, and the functional protein interaction network was obtained through the STRING database. ClusterProfiler and Pathview were used to perform Gene Ontology function analysis and KEGG pathway enrichment analysis of the effective targets of Baihe Decoction in CHD. Finally, we used MOE software for molecular docking of the compounds to their targets.

Results: Fifteen active components of Baihe Decoction in CHD were screened, which corresponded to 145 targets in CHD, including 30 targets with strong correlations. The key targets included Jun, Aktl, MAPK1, RELA, IL6, CXCL8, EGFR, MAPK14, ESR1, and FOS, which were found to play important roles in the treatment of CHD. The results of molecular docking further illustrated the roles that the compounds with quercetin and $\beta$-sitosterol play in the treatment of CHD through their interference with AKT1 and MAPK1 target proteins.

Conclusions: This study has preliminarily revealed the mechanism of Baihe Decoction in the treatment of CHD. The components of TCM may intervene in the processes of CHD occurrence and development by regulating cardiomyocytes and antioxidative stress, and by participating in inflammation and immune response. Moreover, in the clinical syndrome differentiation of TCM, Baihe Decoction can be used as the main drug to treat CHD and angina pectoris due to qi stagnation and blood stasis caused by emotional discomfort.

Keywords: Baihe Decoction; coronary heart disease (CHD); network pharmacology; molecular docking; signaling pathway

Submitted Dec 28, 2020. Accepted for publication Mar 23, 2021.

doi: $10.21037 /$ apm-21-543

View this article at: http://dx.doi.org/10.21037/apm-21-543 


\section{Introduction}

Coronary heart disease (CHD), also termed ischemic heart disease, is globally recognized as a serious, healthendangering disease that brings a substantial socioeconomic burden to patients and their families. Moreover, the morbidity and fatality rates of CHD are on the rise. According to a research paper published by The Lancet in June 2019, vascular disease is $2^{\text {nd }}$ only to stroke as a leading cause of death in China. Between 1990 and 2017, the agestandardized death rate due to ischemic heart disease increased by $20.6 \%$ among the Chinese population (1). At present, traditional Chinese medicine (TCM) is attracting wide attention in the prevention and treatment of cardiovascular diseases due to its attributes of multicomponent, multi-target, and multi-path regulation of the body, and fewer side effects.

"Shi Fang Ge Kuo" by Chen Xiuyuan, a well-known physician in the Qing Dynasty, describes 2 classic prescriptions for chest pain and heartache: Danshen Decoction and Baihe Decoction. These decoctions are widely used in the treatment of angina pectoris and other diseases in clinical practice. Multiple effects of Danshen Decoction on cardiovascular diseases have been evidenced, and Danshen-based drugs have proven extremely effective in the treatment of CHD (2-4). However, Baihe Decoction has been the focus of few research reports. Although the 2 decoctions are designed for heart pain caused by "qi stagnation" and "blood stasis", both have a unique focus. Baihe Decoction consists of 2 medicinal plant extracts: Lily and Wu yao. Chen Xiuyuan stated that when "Longtime pain turns out to be stagnant, Baihe Decoction is suitable" (5). Based on preliminary clinical data, Zhang Lei, a master of TCM in Henan, successfully used Baihe Decoction to treat patients with stubborn symptoms of chest pain and heartache. He explained that Lily can control the "lung qi" and lower the qi of the viscera, promoting the smooth flow of the qi, with no pain: this is key to the effectiveness of Lily in treating heart pain. Baihe Decoction also has the beneficial effects of clearing "heat," promoting qi, and relieving pain, and it is especially suitable for treating chest pain and heartache caused by stagnation of qi and blood stasis $(6,7)$.

However, studies on Baihe Decoction have only explored its clinical use in the treatment of cardiovascular diseases, and the pharmacological mechanism of this TCM has yet to be the focus of a research report. In recent years, network pharmacology has been widely used in TCM researches leading to a number of interesting discoveries $(8,9)$, especially in recent COVID-19 pandemic (10). Therefore, in the present study, using a network pharmacology approach, we screened the effective active ingredients of Baihe Decoction and their targets in CHD, predicted the related targets and signaling pathways, and constructed an active ingredient-target-disease network to explore the therapeutic mechanism of this decoction in CHD. We present the following article in accordance with the MDAR checklist (available at http://dx.doi.org/10.21037/apm-21-543).

\section{Methods}

\section{Selection of chemical components in Baibe Decoction}

We searched for the targets of action of all chemical components of "Lily" and "Wu yao" through the Traditional Chinese Medicine Systems Pharmacology (TCMSP) Database and Analysis Platform (https://tcmspw. com/tcmsp.php). According to the pharmacokinetic rules, as the restrictive screening conditions for obtaining the active ingredients of Lily and $\mathrm{Wu}$ yao, we set the oral bioavailability (OB) threshold at $\geq 30 \%$ and the druglikeness (DL) threshold at $\geq 0.18$ in the parameters of absorption, distribution, metabolism, and excretion (ADME). The study was conducted in accordance with the Declaration of Helsinki (as revised in 2013).

\section{Collecting the target proteins of the active ingredients of Baibe Decoction}

All the target proteins of the active ingredients of Baihe Decoction were collected through the TCMSP database, and the Uniprot protein database (https://www.uniprot. org/) was used to convert the names of the collected target proteins into the ID names of the corresponding genes.

\section{Obtaining the targets of Baibe Decoction in CHD}

The Online Mendelian Inheritance in Man (OMIM, https://www.omim.org/) and the GeneCards (https://www. genecards.org/) databases were searched for CHD gene data. The results from the 2 databases were combined and integrated to obtain the gene target library of CHD. Then, the targets corresponding to the effective active ingredients of Lily and $\mathrm{Wu}$ yao and the target genes associated with CHD were compared. A Venn diagram was drawn and common target genes were screened out as potential 
targets of the active ingredients of Baihe Decoction in the treatment of CHD.

\section{Construction of the drug-component-disease target network}

The active ingredients of Baihe Decoction, their corresponding targets, and the target genes associated with CHD were entered into Cytoscape (version 3.7.1), and a drug-component-disease target network relationship diagram was constructed to clarify the potential interactions between CHD target genes and active ingredients of Baihe Decoction.

\section{Construction of the target protein interaction network}

The target proteins corresponding to the components of Baihe Decoction in CHD were imported into the STRING 11.0 database to construct the protein-protein interaction network (PPI). To obtain the PPI network, we set the species as "Homo sapiens" and the lowest interaction threshold as "combined-score" $\geq 0.9$. The Cytoscape software was used to draw the PPI network visual diagram, and the PPI histogram was drawn using the barplot tool. Then, the top 30 core target protein genes were ordered according to the strength of the protein interaction.

\section{Gene function and patbway enrichment analysis}

The $\mathrm{R}$ language clusterProfiler and pathview packages were used to perform Gene Ontology (GO) functional enrichment analysis and Kyoto Encyclopedia of Genes and Genomes (KEGG) pathway enrichment analysis. The results were presented in the form of a histogram and a bubble chart. The key signaling pathways were screened by the enrichment factor value and enrichment degree, and the mechanism by which Baihe Decoction interferes with biological processes and metabolic pathways in CHD was investigated.

\section{Molecular matching of Baibe Decoction ingredients and key target proteins}

The crystal structures of the candidate proteins bound by the important compounds of Baihe Decoction were downloaded from the Protein Data Bank (PDB) protein structure database (http://www.pdb.org/). To ensure accuracy and consistency, Molecular Operating
Environment (MOE) 2015 software was used to remove ligands and to add hydrogen, remove water, optimize and repair amino acids, and minimize the energy of all candidate targets. MOE was used to test the matching accuracy between Baihe Decoction compounds and candidate target protein, due to its high levels of accuracy and consistency.

\section{Statistical analysis}

All statistical process were concuted in software mentioned before respectively.

\section{Results}

\section{Active ingredients in Baibe Decoction}

A total of 212 chemical constituents of Baihe Decoction were retrieved from the TCMSP database including 84 and 128 chemical constituents for Lily and $\mathrm{Wu}$ yao, respectively. With $\mathrm{OB} \geq 30 \%$ and $\mathrm{DL} \geq 0.18$ as indicators, 16 biologically active ingredients were screened out (Table 1), including 7 active ingredients of Lily and 9 active ingredients of $\mathrm{Wu}$ yao. Among the chemical ingredients, beta-sitosterol was found in both Lily and Wu yao. By combining ADME characteristics, we identified 15 chemical components in Baihe Decoction with good bioavailability. The analysis of the drug composition of Lily showed that, chemically, it is mainly composed of steroidal saponins, such as stigmasterol, $\beta$-sitosterol, and cholesstanol glycosides (26-O- $\beta$-D)glu-3 $\beta$, 26-dihydroxy cholestan-16, 22-dioxo-3-O- $\alpha$-rha $(1 \rightarrow 2)-\beta$-D-glucoside; $26-\mathrm{O}-\beta-\mathrm{D}$-glu-3 $\beta$, 26-dihydroxy-5cholesten-16, 22-dioxo-3-O- $\alpha$-rha ( $1 \rightarrow 2)$ - $\beta$-D-glucoside). For $\mathrm{Wu}$ yao, flavonoids (quercetin and nubigenol), alkaloids (norboldine and boldine), terpenoid lactones (Agropyrone C09495), and sitosterol were found to be the active ingredients.

\section{Predicted target genes of Baibe Decoction}

A total of 5,552 CHD-related target genes were obtained through searches of the OMIM and GeneCards databases. Following deduplication, 5,282 target genes associated with CHD were finally obtained. The potential target genes of the drugs in Baihe Decoction were matched with the CHD-related genes, and Venn 2.1 software was used to draw a Venn diagram, which is shown in Figure 1. A total of 145 CHD-related target genes were identified for Baihe Decoction, accounting for $86.8 \%$ of the total targets of 
Table 1 Active compounds in Baihe Decoction

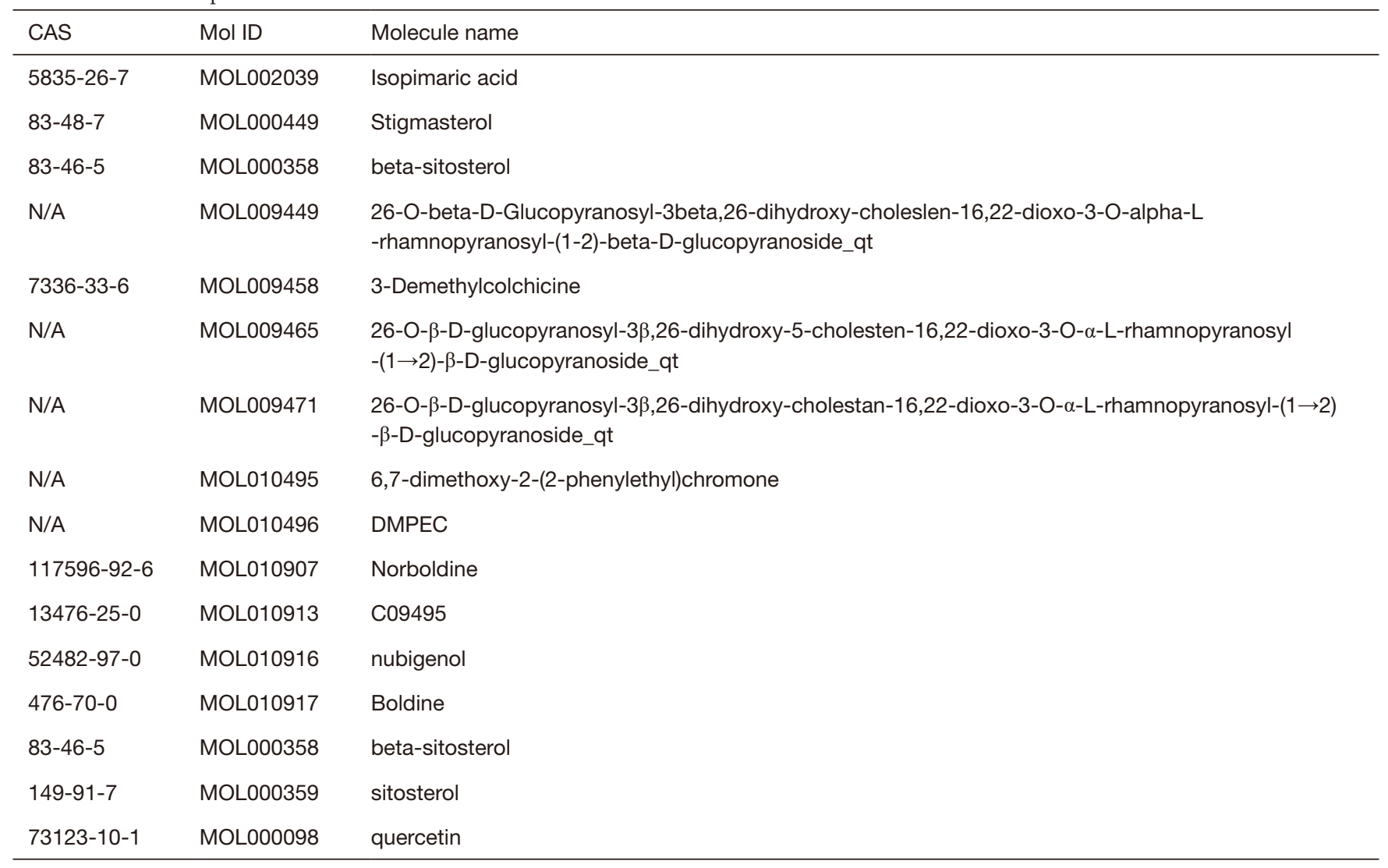

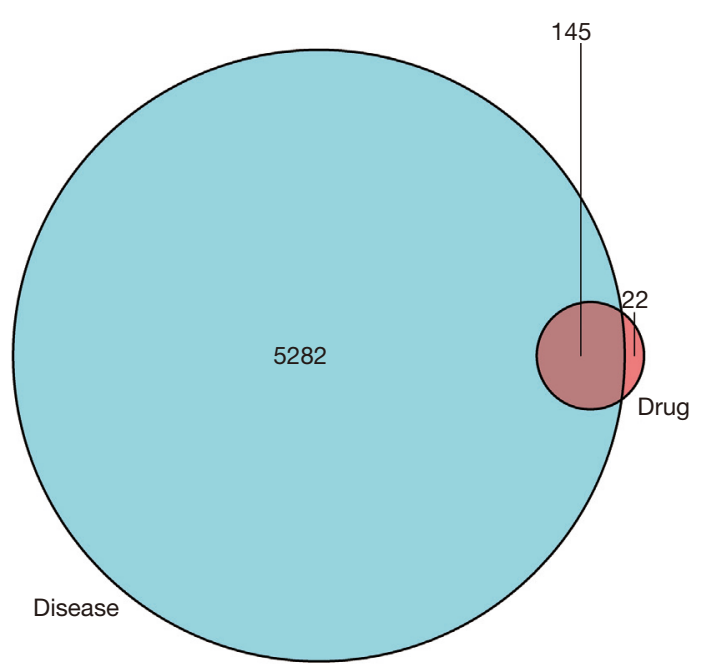

Figure 1 Venn map of intersection between CHD and Baihe Decoction targets. CHD, coronary heart disease. active ingredients of this TCM. This result indicated Baihe Decoction to have a strong effect on CHD, as well as a high research value.

\section{Network construction and analysis of active ingredients in Baibe Decoction and CHD}

The active ingredients of Baihe Decoction and the corresponding target proteins of CHD were imported into Cytoscape, and a visual network diagram was constructed (Figure 2). The network comprised 162 nodes, 145 target proteins corresponding to the disease, and 267 edges. The results showed that the 15 effective components of Baihe Decoction, corresponding to CHD-related genes, including RXRA, PGR, PTGS2, NCOA2, NCOA1, NR3C2, ADH1C, PTGS1, ADRA2A, SLC6A2, ADRB2, AKR1B1, 


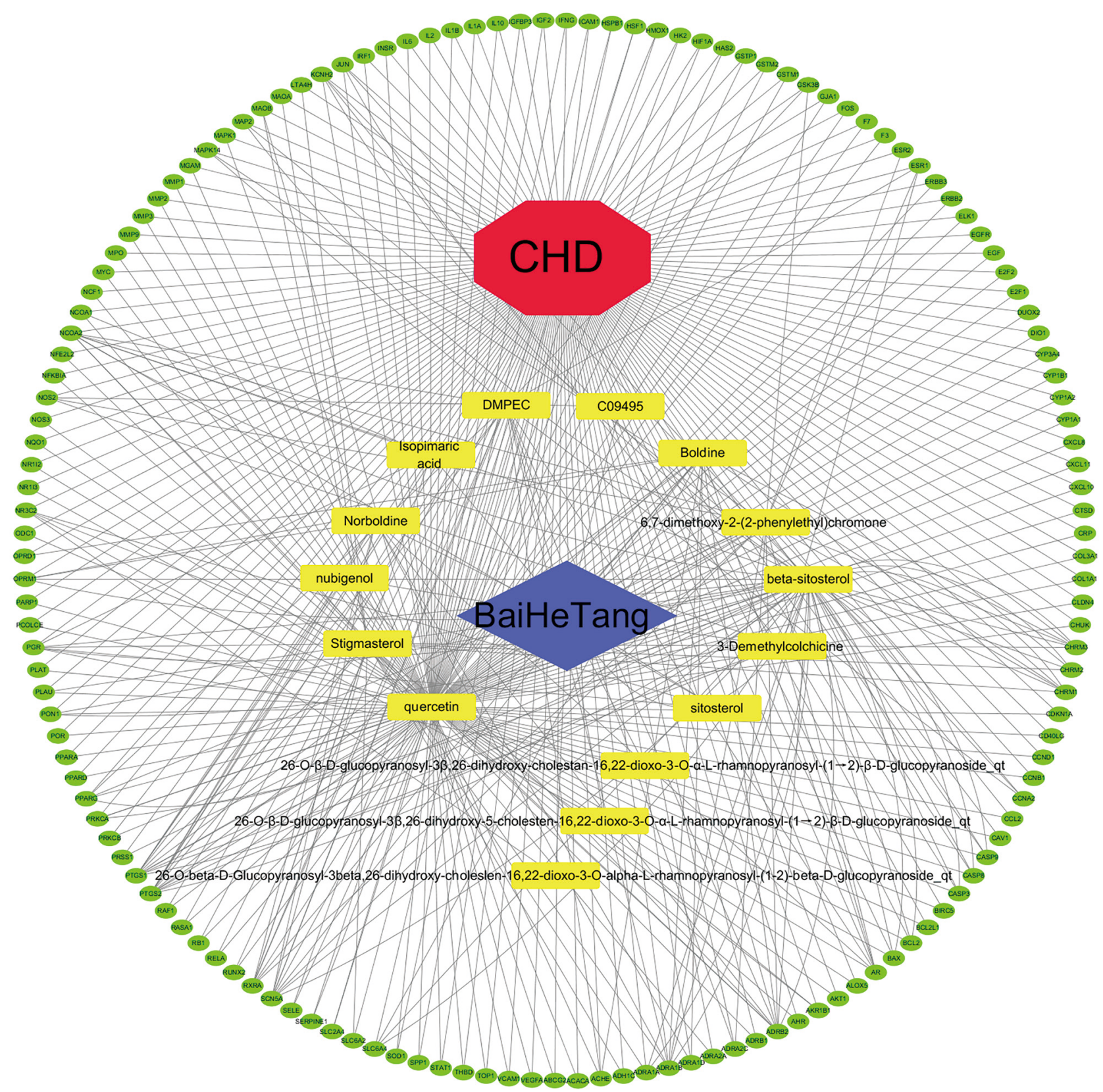

Figure 2 Effective component-target-disease gene network. Red represents CHD, purple represents Baihe Decoction, yellow represents active ingredients of Baihe decoction, and green represents the target points of Baihe Decoction for CHD. The connected edges represent the relationships between the active ingredients of the drugs in Baihe Decoction and the CHD-related targets. CHD, coronary heart disease. 


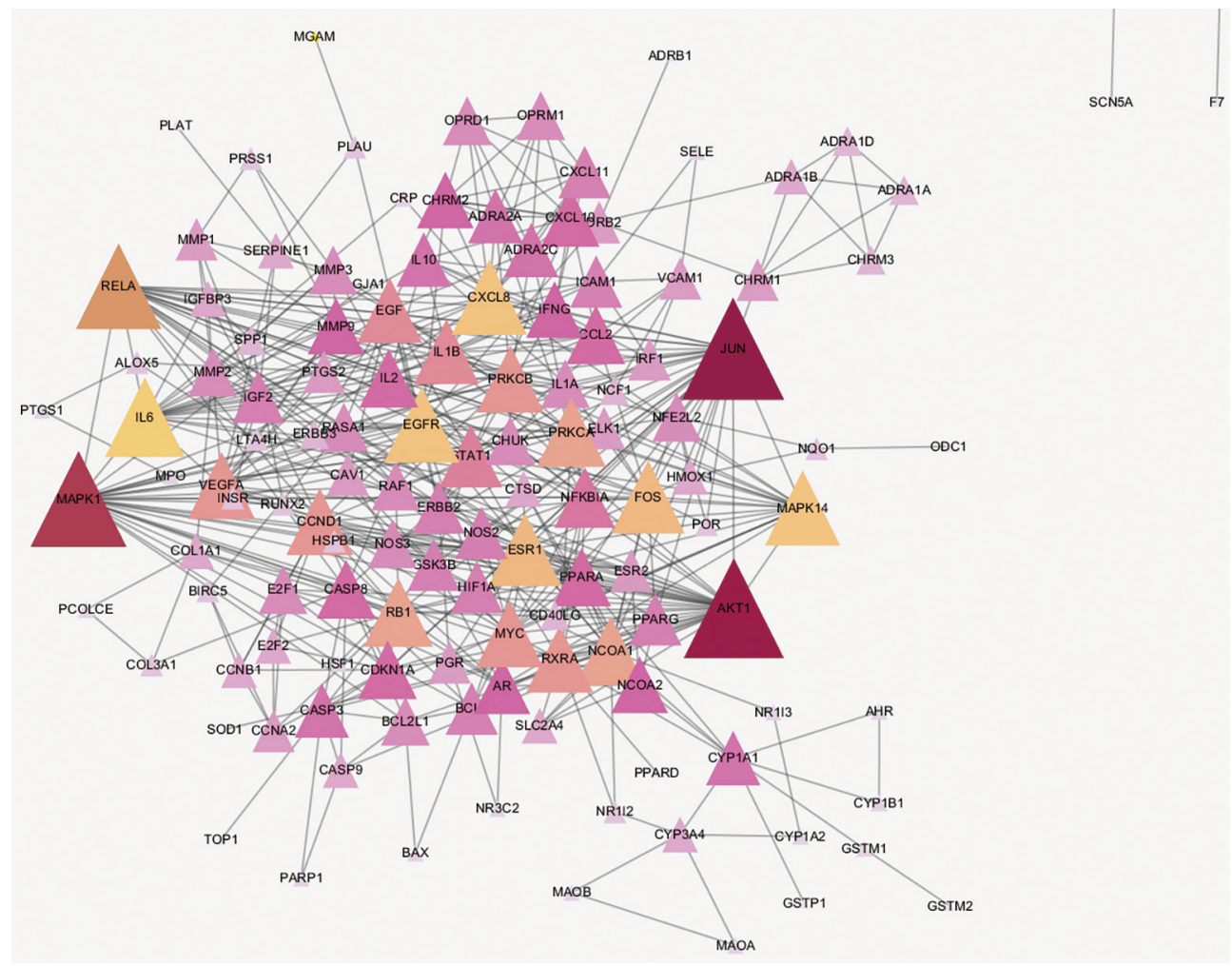

Figure 3 Protein-protein interaction network of Baihe Decoction

PLAU, LTA4H, MAOB, MAOA, 145 target genes such as CHRM3, CHRM1, ADRB1, SCN5A, and ADRA1A.

\section{Analysis of the Baibe Decoction Gene Protein Interaction Network}

The 145 target proteins of Baihe Decoction in CHD were imported into the STRING database and then the PPI data were imported into Cytoscape software. A protein relationship network diagram was drawn (Figure 3), and the top 30 target genes were arranged according to the enrichment degree. They included the proto-oncogene Jun, serine/threonine kinase 1 (Aktl), mitogen activated protein kinase 1 (MAPK1), v-rel reticuloendothelial hyperplasia virus oncogene homolog A (RELA), interleukin-6 (IL6), interleukin-8 (CXCL8), epidermal growth factor receptor (EGFR), mitogen activated protein kinase 14 (MAPK14), estrogen receptor (ESR1) and proto-oncogene FOS, respectively. At the same time, the PPI histogram in Figure 4 revealed the Jun, Aktl, MAPK1, RELA, and IL6 genes to have more than 20 adjacent nodes, indicating that these genes have important interactions in the protein network.

\section{GO biological functional analysis of Baibe Decoction}

GO functional analysis of the key targets of Baihe Decoction in the treatment of CHD was performed, including biological processes (BPs), molecular functions (MFs), and cellular components (CCs). The top $20 \mathrm{BPs}$ with the smallest $\mathrm{P}$ value were analyzed. In terms of BPs (Figure 5A), the targets of Baihe Decoction in CHD were mainly enriched in lipopolysaccharide reaction, bacterial source molecular reaction, oxygen level reaction, reactive oxygen metabolism, steroid hormone reaction, nutrient level reaction, oxygen deficiency response, oxidative stress response, blood circulation, and circulatory system processes. In terms of MFs (Figure $5 B$ ), the targets were mainly involved in nuclear receptor activity, DNA binding transcription activation activity, steroid hormone receptor activity, adrenaline receptor activity, cytokine receptor binding, heme binding, RNA polymerase II transcription factor binding, and $\mathrm{G}$ protein-coupled amine receptor activity. In terms of CCs (Figure 5C), the targets were mainly enriched in the plasma membrane, mitochondria, RNA polymerase II transcription factor complex, nuclear 


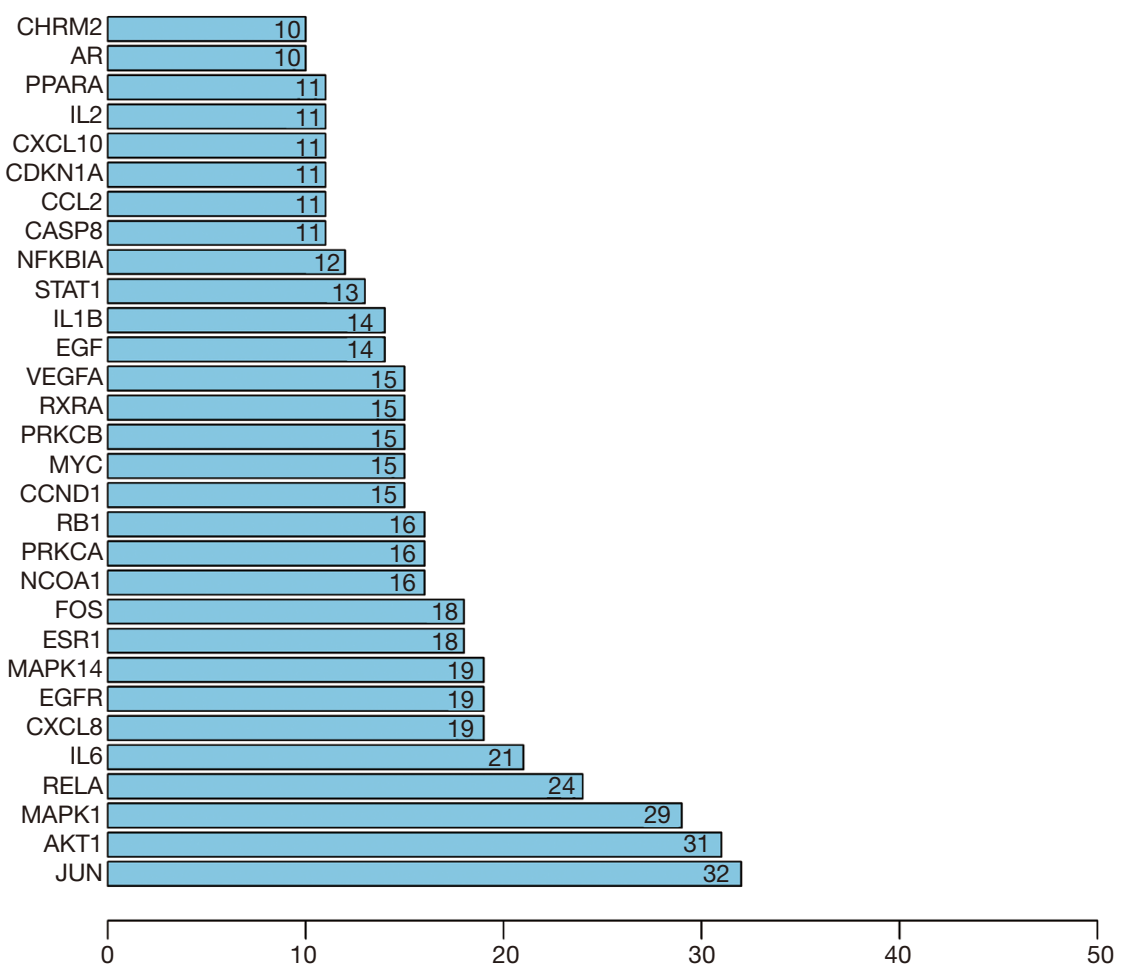

Figure 4 Key target genes of Baihe Decoction in the treatment of coronary heart disease

transcription factor complex, nuclear chromatin, and protein kinase complex. These results suggested that Baihe Decoction plays a therapeutic role CHD by regulating multiple complex BPs.

\section{KEGG pathway enrichment analysis}

KEGG pathway enrichment analysis (Figure 6 and Table 2) was carried out to screen the signaling pathways in which the targets of Baihe Decoction are most significantly enriched. The top 15 most enriched signaling pathways were all significantly correlated to CHD. The most enriched pathway was the phosphatidylinositol 3-kinase/ protein kinase (PI3K-Akt) B signaling pathway, followed by the MAPK signaling pathway, the hypoxia-inducible factor 1 (HIF-1) signaling pathway, endocrine resistance, cellular senescence, apoptosis, the Toll-like receptor signaling pathway, the C-type lectin receptor signaling pathway, the calcium signaling pathway, the ErbB signaling pathway, and the NF- $\mathrm{KB}$ signaling pathway.

\section{Molecular matching results}

To further verify the candidate compounds for the Baihe Decoction targets in CHD, we tested the matching accuracy of quercetin and beta-sitosterol with AKT1 (PDB: 4GV1), and MAPK1 (PDB: 6OPH). These target proteins were chosen for study due to their key positions in the PPI network and their participation in key pathways, which suggested that they may play a key role in the response of CHD to Baihe Decoction compounds. The best matching attitude is the root mean square deviation (RMSD) between the predicted conformation and the observed X-ray crystal conformation reach the lowest limit. A model with RMSD $\leq 4 \AA$ is considered reliable, and a model with RMSD $\leq 2 \AA$ is considered accurate. The final score of the overall evaluation is E_score2. The smaller the corresponding value, the smaller the binding energy between molecules, which reflects better binding between the 2 (generally less than -5).

We also mapped the three-dimensional conformation of the matching of quercetin and $\beta$-sitosterol with AKT1 and 
A

Response to lipopolysaccharide Response to molecule of bacterial origin

Response to oxygen levels

Reactive oxygen species metabolic process

Response to steroid hormone

Response to nutrient levels

Response to hypoxia

Response to decreased oxygen levels

Response to reactive oxygen species

Response to oxidative stress

Response to antibiotic

Response to metal ion

Response to ketone

Blood circulation

Circulatory system process

Cellular response to drug

Cellular response to oxidative stress

Reactive oxygen species biosynthetic process

Cellular response to reactive oxygen species Regulation of body fluid levels

B

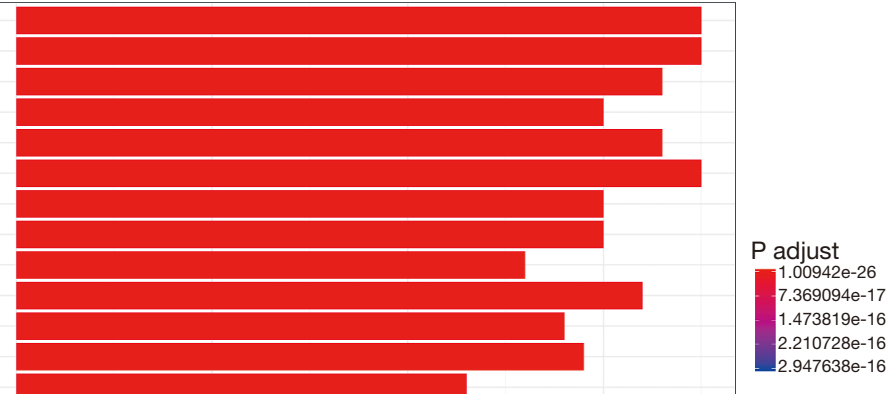

Transcription factor activity, direct ligand regulated sequence-specific DNA binding

Steroid hormone receptor activity

Adrenergic receptor activity

Protein-coupled amine receptor activity RNA polymerase II transcription factor binding

Cytokine receptor binding Heme binding

DNA-binding transcription activator activity,RNA polymerase II-specific

Tetrapyrrole binding

Cytokine activity

biquitin-like protein ligase binding

Nuclear receptor transcription coactivator activity

Kinase regulator activity

Nuclear hormone receptor binding

Antioxidant activity

Oxidoreductase activity, acting on paired donors, with incorporation or reduction of molecular oxygen

Ubiquitin protein ligase binding

Repressing transcription factor binding

Protein kinase regulator activity

C

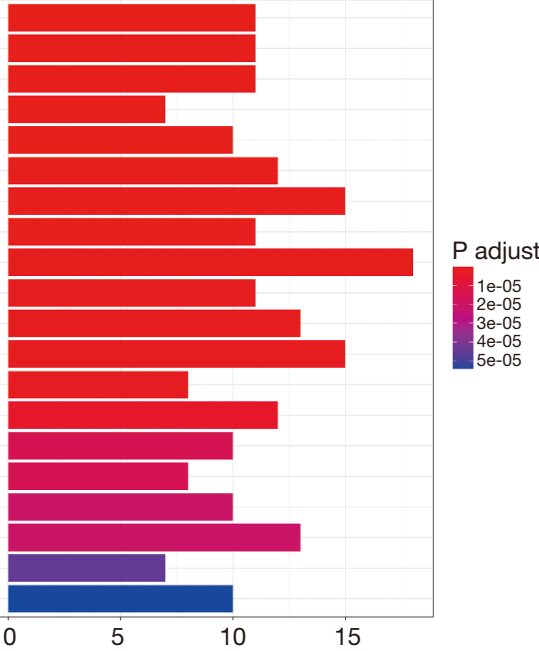

Membrane raft Membrane microdomain Membrane region Caveola

Plasma membrane raft RNA polymerase II transcription factor complex

Transcription factor complex Nuclear transcription factor complex

Nuclear chromatin

Mitochondrial outer membrane Integral component of presynaptic membrane. Intrinsic component of presynaptic membrane. Organelle outer membrane Integral component of postsynaptic membrane Outer membrane

Intrinsic component of postsynaptic membrane. Cyclin-dependent protein kinase holoenzyme complex Protein kinase complex. Integral component of synaptic membrane Presynaptic membrane

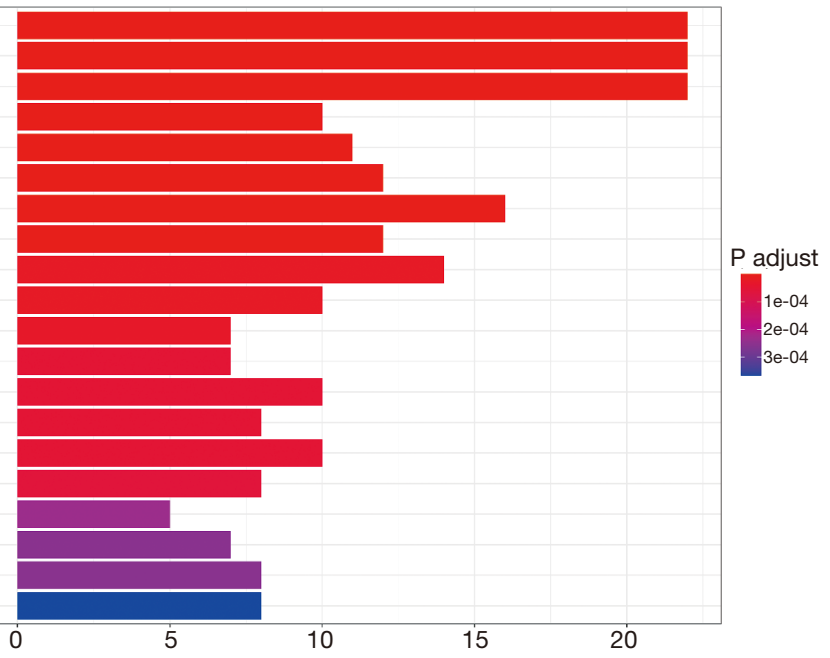

Figure 5 Gene Ontology enrichment analysis of the key targets of Baihe Decoction in CHD. (A) Gene Ontology biological processes; (B) Gene Ontology molecular functions; (C) Gene Ontology cellular components. CHD, coronary heart disease. 


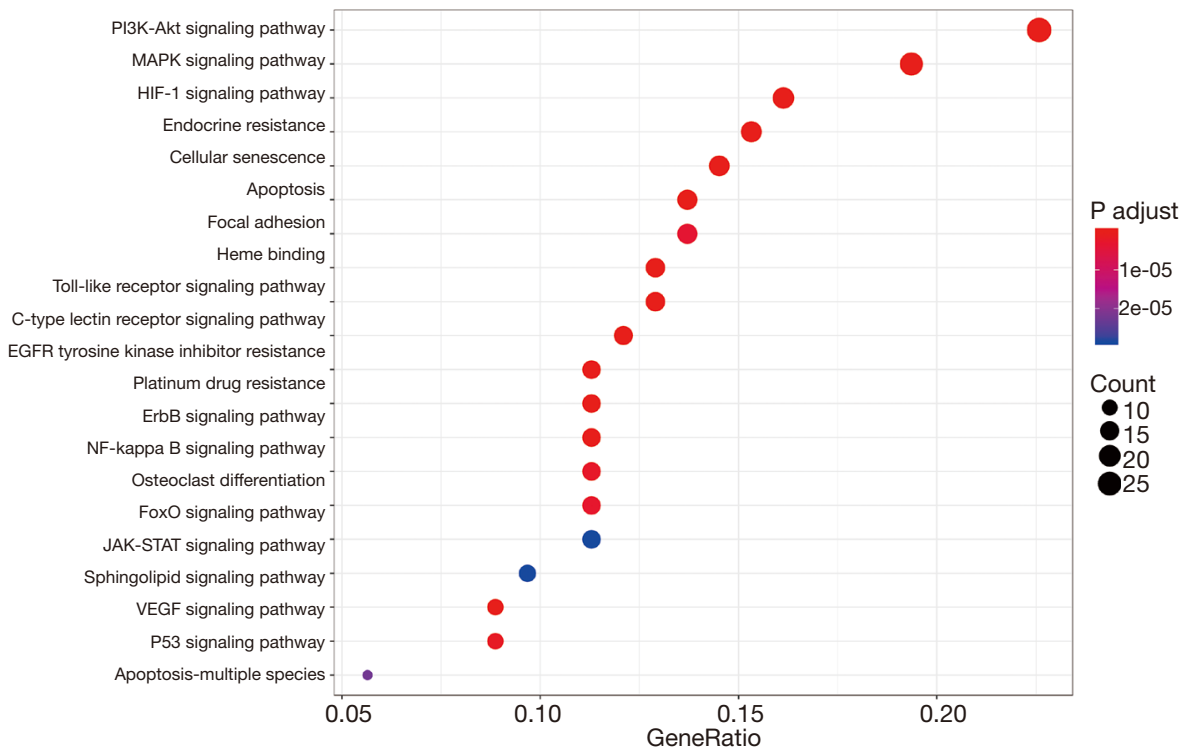

Figure 6 Kyoto Encyclopedia of Genes and Genomes pathway enrichment analysis of Baihe Decoction targets.

Table 2 Top signaling pathways of Baihe Decoction targets

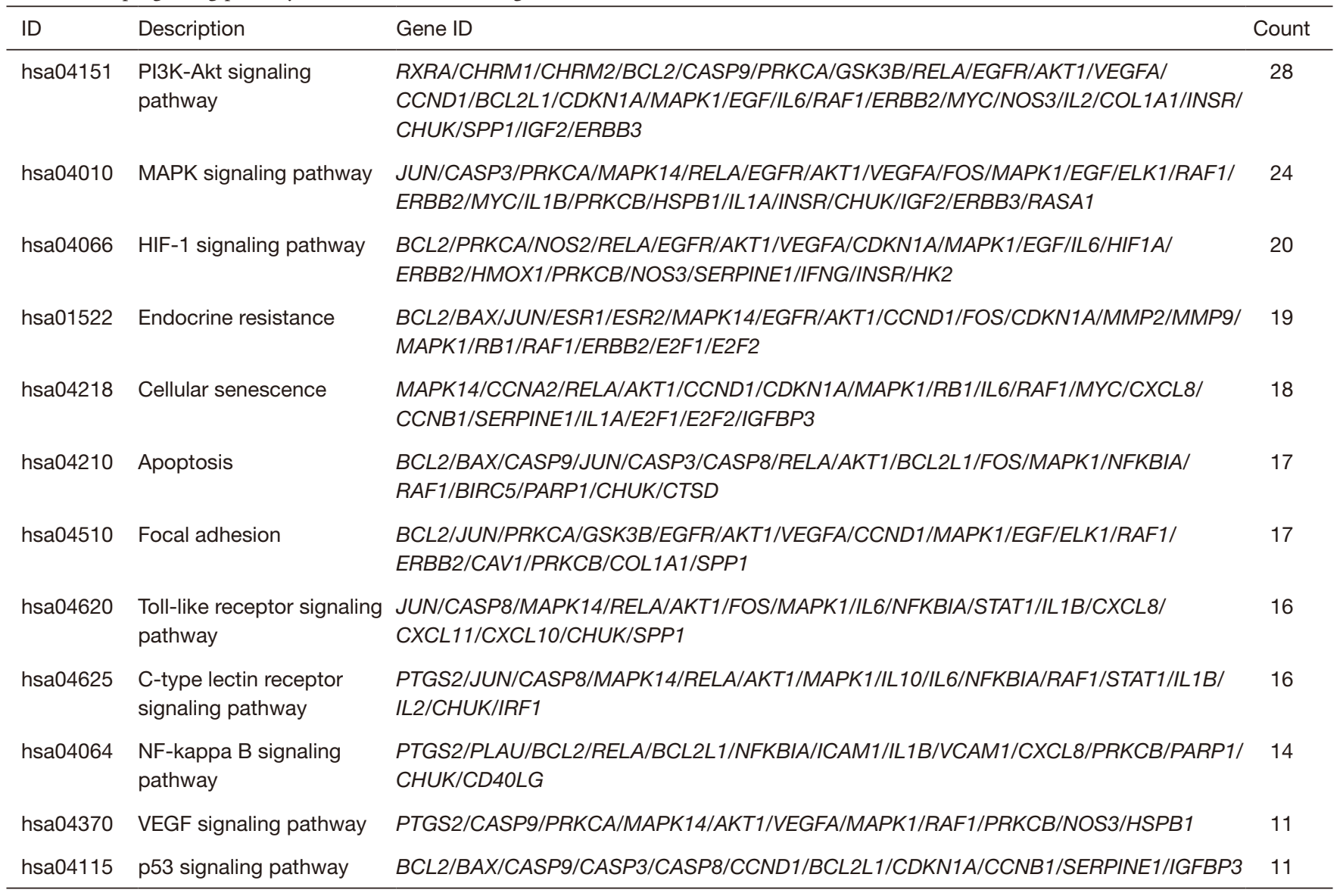



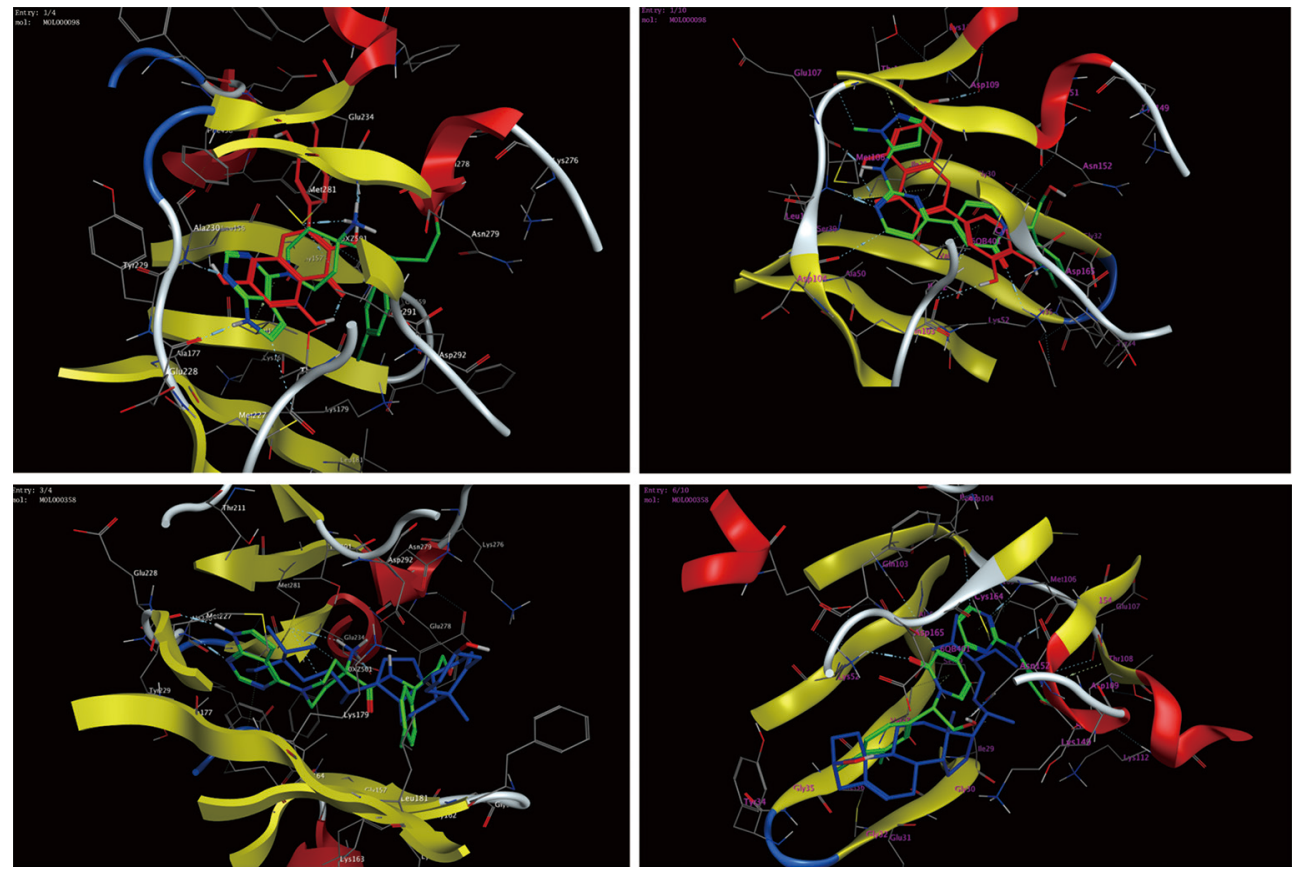

Figure 7 Molecular docking pattern of quercetin and beta-sitosterol with AKT1 (left) and MAPK1 (right).

Table 3 Binding energies of main active ingredients of Baihe Decoction and AKT1 (PDB:4GV1) and MAPK1 (PDB:6OPH)

\begin{tabular}{lcccccccc}
\hline Mol & PDB_structure & S & rmsd_refine & E_conf & E_place & E_score1 & E_refine & E_score2 \\
\hline Beta-sitosterol & MAPK1_6OPH & -7.5266275 & 1.729784 & 51.6824 & -57.263298 & -8.9986725 & -34.89711 & -7.5266275 \\
Beta-sitosterol & AKT1_4GV1 & -7.8366866 & 1.7354221 & 50.203396 & -59.053967 & -9.4693298 & -37.883968 & -7.8366866 \\
Quercetin & MAPK1_6OPH & -6.532742 & 10258478 & 3.1375182 & -110.89886 & -14.375526 & -35.232117 & -6.532742 \\
Quercetin & AKT1_4GV1 & -6.5123148 & 1.4737077 & 0.17133738 & -93.769257 & -13.425066 & -31.620445 & -6.5123148 \\
\hline
\end{tabular}

MAPK1 (Figure $7 A, B$ ), and marked the structure of small molecules with different colors. A structural space between the 2 maps where there were larger repeat regions could be observed, proving that there was great similarity between the conformation of the small molecule and the ligand. The results of the matching analysis showed that the scores of the target proteins and the corresponding compound molecule were less than -5 , indicating that the target proteins and the compound molecules had good binding properties (Table 3). The matching analysis successfully predicted that quercetin and $\beta$-sitosterol would bind well to the 2 active sites of the tested target proteins.

\section{Discussion}

CHD is caused by atherosclerosis of coronary arteries and can lead to myocardial ischemia, hypoxia, and even necrosis. It mainly manifests as angina, myocardial infarction, arrhythmia, and heart failure (8). In recent years, studies have confirmed that interventions with TCM can reduce the incidence of acute adverse cardiovascular events among patients with CHD (9). TCM symptom differentiation and treatment plays an indispensable role in preventing and treating this disease; however, it also faces challenges in the era of precise medicine. With the rapid progress of network pharmacology, Chinese medicine research also made significant development. Many classical Chinese medicine regimens have been deeply studied and outstanding results have been reached $(8,9)$.

In this study, we found that the effective ingredients of Baihe Decoction, Lily and Wu yao, mainly contain sterols, saponins, alkaloids, and flavonoids. Modern 
pharmacology has shown that Lily has antioxidative, antiinflammatory, anti-depression, anti-tumor, hypoglycemic, anti-stress damage, immune regulatory, and other biological activities (11). It can also inhibit the body's absorption of cholesterol, promote cholesterol degradation, lower plasma triglyceride levels, and prevent and treat coronary atherosclerosis (12-14). Wu yao has anti-inflammatory, analgesic, anti-tumor, and antioxidative effects, and can lower blood pressure, regulate gastrointestinal motility, protect the liver, excite the myocardium, and regulate blood coagulation (15). A study by Han (16) focusing on quercetin, as the active ingredient with the highest content of flavonoids in $\mathrm{Wu}$ yao, concluded that quercetin has good antioxidant and blood stasis-removing effects, which is of great significance for treating cardiovascular diseases. By conducting in vivo experiments, Chen et al. (17) found that quercetin can upregulate the expression of NF- $\mathrm{KB}$ p 65 and TLR2 protein, promote the recovery of immune organ function, and increase the body's antioxidant levels, as well as improve the immune function of mice. Han et al. (18) found that the effective components of $\mathrm{Wu}$ yao leaves can block the TLR-4/NF- $\kappa B$ signaling pathway and play a role in lowering lipids and liver protection.

Domestic studies have also proposed the possible existence of an evolutionary law of the occurrence and development of CHD. The proportion of patients with qi stagnation after intervention for CHD is significantly increased. Major surgery and other factors can result in mental trauma for patients (19), and more than $30 \%$ of patients with CHD have depressive symptoms (20). Lily can relieve the symptoms of depression and anxiety by increasing the content of serotonin and dopamine, thus improving monoamine neurotransmitter insufficiency, reducing the levels of hypothalamic corticotropin-releasing factor, and increasing the pathway of hippocampal glucocorticoid receptor messenger RNA. The effective ingredients of Lily, including $26-\mathrm{O}-\beta-\mathrm{D}$-glu- $3 \beta$, 26-dihydroxy- $\Delta-5$-choleslen16-22-dioxo-3-O- $\alpha$-L-rhamnopyranosyl-( $1 \rightarrow 2)-\beta$-Dglucopyranoside, are all anti-depressant substances $(21,22)$. $\mathrm{Wu}$ yao has the functions of soothing the liver, regulating qi, promoting qi, and relieving depression. The alkaloids in $\mathrm{Wu}$ yao can bind to nicotinic acetylcholine receptors by regulating the release of dopamine neurotransmitters, and act on ADPH1, CDK2B, and DA protein targets to motivate qi and relieve pain (15). According our research results, Baihe Decoction may be a valuable treatment for CHD accompanied by depression. It can be used as the main medicine to treat depressive symptoms associated with interventional surgery and previous myocardial infarction.

In this study, through the analysis of PPI protein interactions, the main targets of Baihe Decoction were found to be Jun, Aktl, MAPK1, RELA, IL6, CXCL8, EGFR, MAPK14, ESR1, and FOS, which act on RXRA, PGR, PTGS2, NCOA2, NCOA1, NR3C2, ADH1C, PTGS1, ADRA2A, SLC6A2, ADRB2, AKR1B1, and other target genes. These core target proteins participate in multiple inflammation, immunity, metabolism, and cell proliferation-related signaling pathways to treat CHD. Jun and FOS are oncoproteins which have the functions of transcription, and regulation of cell growth and metabolism. They can also form FOS-Jun complexes, which can regulate the expression of IL- 6 and IL- $1 \beta$, as well as inflammatory responses. FOS-Jun complexes is effective in myocardial ischemia, myocardial infarction, and postoperative restenosis, and plays an important role in the pathogenesis of various cardiovascular systems (23). Akt1 is 1 of serine/ threonine protein kinases which regulates inflammation, immunity, and cell metabolism via signaling pathways including P13K-Akt and MAPK. Studies analyzing the GSE18612 and GSE64554 gene maps of epicardial adipose tissue of patients with CHD concluded that the AKT1 gene can serve as 1 of the main biomarkers and therapeutic targets of CHD (24). MAPK1 and MAPK14 are members of the MAPK family, which can phosphorylate and activate the expression of transcription factors and downstream genes, and play a role in the regulation of cell survival and death, as well as inflammation (25). RELA is a transcription factor of the NF- $\mathrm{KB}$ family which can regulate cell adhesion, infiltration, metastasis, and numerous other processes. Its enhanced expression can promote the occurrence and development of atherosclerosis (26). IL6 and CXCL8 are both pro-inflammatory cytokines which are involved in the pathological process of inflammation as regulators of inflammatory response. Through a comparison of the biological network characteristics of angina pectoris of CHD with Qi deficiency and blood stasis syndrome, and Qi stagnation and blood stasis syndrome (27), Liu found that EGFR, IL6, AR, CHRM2, PPARA, IL2, EGF, and ESR1, which were identified as key targets of Baihe Decoction in this study, were the key genes of Qi stagnation and blood stasis syndrome. Therefore, using Baihe Decoction in the treatment of CHD caused by qi stagnation and blood stasis would be reasonable. This finding is highly consistent with clinical application and, to a certain extent, reflects the role of network pharmacology in predicting the mechanism of drug action. 
GO and KEGG enrichment analyses found that Baihe Decoction mainly regulates nuclear receptor activity, DNA binding transcription activation activity, steroid binding, cytokine receptor binding, heme binding, and RNA polymerase II transcription factor binding to participate in coronary artery inflammation and immune responses (28), such as lipopolysaccharide response, bacterial molecular source response, oxidative stress response, steroid hormone response, and other biological processes related to CHD. Its mechanism of action mostly involves anti-inflammatory and antioxidative stress effects, to which the PI3K-Akt, MAPK, and HIF-1 signaling pathways are closely related. The PI3K-Akt signaling pathway is an important signal transduction pathway that regulates cardiac function mainly by activating PI3K, binding downstream Akt, phosphorylating Akt, initiating downstream target gene transcription, and regulating the growth and survival of cardiomyocytes and blood vessels. Regeneration and maintenance of cardiac systolic function, regulation of signal transduction, and induction of cell autophagy all affect the atherosclerotic process (29). Ren et al. (30) observed that sphingosine 1-phosphate can effectively inhibit cardiomyocyte apoptosis in CHD rats by regulating the expression of related proteins in the PI3K/ Akt signaling pathway. The MAPK signaling pathway transmits signals from the cell membrane to the nucleus in response to various stimuli, and also participates in the signal transduction of various growth factors, cytokines, mitogens, and hormone receptors after activation. It plays an important regulatory role in cell growth and apoptosis, inflammation, and stress response $(31,32)$. Zuo et al. (33) reported that genetic polymorphisms in MAPK genes may affect blood lipid metabolism and coagulation function in CHD, and participate in inflammatory reactions. HIF-1, as part of the HIF-1 signaling pathway, acts as the main regulator of oxygen homeostasis and is extremely important to the body's response to low oxygen levels or hypoxia. It is a key regulator of stimulation not only in myocardial hypoxia but also in myocardial stress (34). Studies such as that by Zhang et al. (35) found that the HIF-1 signaling pathway upregulates vascular endothelial growth factor, nitrous oxide, reactive oxygen species, and platelet-derived growth factor by acting on the specific response of vascular endothelial cells, smooth muscle cells, and macrophages, subsequently resulting in endothelial cell dysfunction, proliferation, angiogenesis, and inflammation. Targeting HIF-1 may become 1 of the key therapeutic strategies for coronary atherosclerosis (36). However, in brief, drug innovation and research through network pharmacology include several steps: network construction, network analysis and network verification; then experimental studies and clinical researches are warranted to confirm the results of network pharmacology. The major difficulty is the multiple possible targets derived from network pharmacology. How to select the best one remains a challenge for investigators.

In conclusion, this study has provided theoretical evidence that Baihe Decoction may interfere with the occurrence and development of CHD by regulating cardiomyocyte proliferation, transcription, and apoptosis, protecting against oxidative stress damage, and participating in inflammation and immune responses and other pathways. The PI3K-Akt, MAPK, HIF-1 signaling pathways may be the most important pathways involved in the effects of Baihe Decoction on CHD. Preliminarily, this study speculates that the effective active ingredients of Baihe Decoction convert extracellular signals into cellular responses through receptor tyrosine kinase (RTK), and activate and promote the phosphorylation of Akt, thereby regulating RTKPI3K-AKT signaling pathways, which plays a role in CHD. The results of molecular matching also showed that the compounds quercetin and $\beta$-sitosterol in Baihe Decoction exert their therapeutic effects by interfering with the target proteins of AKT1 and MAPK1, proving that these 2 proteins act as the target proteins of quercetin and $\beta$-sitosterol in CHD. Sterol has high credibility as a therapeutic target. Overall, our results provide further evidence for the use of Baihe Decoction in the treatment of CHD. However, this study has some limitations. First of all, pharmacodynamic markers of the regulatory effects of Baihe Decoction on CHD need to be further verified by experimental studies. Second, based on the present results, it is hard to determine the main mechanism involved in the effect of Baihe Decoction on CHD and there is still a lot work to do.

\section{Acknowledgments}

Funding: This research was supported by Henan Zhongjing Prescription and Medicine Modernization Engineering Research Center (Engineering Laboratory) Prevention and Treatment of Cardiovascular and Cerebrovascular Disease Mechanism Research Project (Yu Fa Gai Gao Ji [2017] No. 911), and Henan Province 2020 Science and Technology Research Project (Project no. 202102310173). 


\section{Footnote}

Reporting Checklist: The authors have completed the MDAR checklist. Available at http://dx.doi.org/10.21037/apm21-543

Conflicts of Interest: All authors have completed the ICMJE uniform disclosure form (available at http://dx.doi. org/10.21037/apm-21-543). The authors have no conflicts of interest to declare.

Ethical Statement: The authors are accountable for all aspects of the work in ensuring that questions related to the accuracy or integrity of any part of the work are appropriately investigated and resolved. The study was conducted in accordance with the Declaration of Helsinki (as revised in 2013).

Open Access Statement: This is an Open Access article distributed in accordance with the Creative Commons Attribution-NonCommercial-NoDerivs 4.0 International License (CC BY-NC-ND 4.0), which permits the noncommercial replication and distribution of the article with the strict proviso that no changes or edits are made and the original work is properly cited (including links to both the formal publication through the relevant DOI and the license). See: https://creativecommons.org/licenses/by-nc-nd/4.0/.

\section{References}

1. Zhou M, Wang H, Zeng X, et al. Mortality, morbidity, and risk factors in China and its provinces, 1990-2017: a systematic analysis for the Global Burden of Disease Study 2017. Lancet 2019;394:1145-58.

2. Yan KP, Guo Y, Xing Z, et al. Dan-Shen-Yin protects the heart against inflammation and oxidative stress induced by acute ischemic myocardial injury in rats. Exp Ther Med 2012;3:314-8.

3. Kachur S, Chongthammakun V, Lavie CJ, et al. Impact of cardiac rehabilitation and exercise training programs in coronary heart disease. Prog Cardiovasc Dis 2017;60:103-14.

4. Su BH. Explore the mechanism of danshen on coronary heart disease based on network pharmacology. J Hainan Med Univ 2020;5:340-6.

5. Lin HG. Chen Xiuyuan Medical Book. Beijing: China Press of Traditional Chinese Medicine, 1999:669.

6. Zhang L. Zhang Lei's Clinical Experience Collection.
Beijing: People's Military Medical Press 2008:84-85,99-100.

7. Zhang L. Zhang Lei Medical Book. Henan: Henan Science and Technology Press 2017:661.

8. Zhang GX, Zhang YY, Zhang XX, et al. Different network pharmacology mechanisms of Danshen-based Fangjis in the treatment of stable angina. Acta Pharmacol Sin 2018;39:952-60.

9. Liao JQ, Shi ZX, Du JX. Modern research progress and thinking on the prevention and treatment of coronary heart disease with traditional Chinese medicine. Chin J Tradi Chin Med 2018;11:4813-6.

10. Yu S, Wang J, Shen H. Network pharmacology-based analysis of the role of traditional Chinese herbal medicines in the treatment of COVID-19. Ann Palliat Med 2020;9:437-46.

11. Luo LM, Pei G, Qin L, et al. Research progress on chemical constituents and pharmacological effects of Lily. N Chin Med Clin Pharm 2017;6:824-37.

12. Cofán M, Ros E. Clinical Application of Plant Sterol and Stanol Products. J AOAC Int 2015;98:701-6.

13. Taha DA, Wasan EK, Wasan KM, et al. Lipid-lowering Activity of Natural and Semi-Synthetic Sterols and Stanols. J Pharm Pharm Sci 2015;18:344-67.

14. Rideout TC, Marinangeli CPF, Harding SV. TriglycerideLowering Response to Plant Sterol and Stanol Consumption. J AOAC Int 2015;98:707-15.

15. Deng GM, Xiang B, Xiao XQ. Study on the pharmacodynamics of the main chemical constituents of Wu Medicine based on network pharmacology. Chin Herb Med 2018;21:2147-51.

16. Han HT. Pharmacodynamic evaluation and mechanism of quercitrin in the treatment of cardiovascular diseases. Zhejiang: Zhejiang University, 2017:7-8.

17. Chen LY, Zhou YY, Xu AL, et al. The immunomodulatory effect of quercetin on immunocompromised mice. Chin J Immu 2019;8:2343-9.

18. Han YD, Ye K, Huang JJ, et al. Effects of extracts of black medicine leaves on liver histopathology and TLR-4/NF$\kappa \mathrm{B}$ signaling pathway in hyperlipidemia rats. N Chin Med 2020;15:15-8.

19. Wang CC, Yang Y, Wu S, et al. Study on the evolution of TCM syndromes in different stages of coronary heart disease. Chin J Trad Chin Med 2019;5:2101-6.

20. Rhead JC. How to Change Your Mind: What the New Science of Psychedelics Teaches Us about Consciousness, Dying, Addiction, Depression, and Transcendence. J Psychoactive Drugs 2018;50:460.

21. Chia XS, Wanga SJ, Balochb Z, et al. Research progress on 
classical traditional Chinese medicine formula Lily Bulb and Rehmannia Decoction in the treatment of depression. Biomed \& Pharmaco 2019;5:1-6.

22. Li Y, Miao MS. Chemical pharmacology and clinical application analysis of lily. Chin Med J 2015;7:1021-3.

23. Matsumoto Y, Komatsu K, Shimazu Y, et al. Effect of resveratrol on c-fos expression of rat trigeminal spinal nucleus caudalis and C1 dorsal horn neurons following mustard oil-induced acute inflammation. Eur J Oral Sci 2017;125:338-44.

24. Wang W, Liu Q, Wang Y, et al. Integration of Gene Expression Profile Data of Human Epicardial Adipose Tissue from Coronary Artery Disease to Verification of Hub Genes and Pathways. Biomed Res Int 2019;2019:8567306.

25. Kumar A, Singh UK, Kini SG, et al. JNK pathway signaling: a novel and smarter therapeutic targets for various biological diseases. Future Med Chem 2015;7:2065-86.

26. Harrold AP, Cleary MM, Bharathy N, et al. In vitro benchmarking of NF- $\kappa \mathrm{B}$ inhibitors. Euro J Phar 2020;5:162-78.

27. Liu JW. Preliminary study on biological network characteristics of Qi deficiency and blood stasis syndrome and Qi stagnation and blood stasis syndrome in angina pectoris of coronary heart disease. Beijing: Beijing University of Traditional Chinese Medicine, 2015:45-7.

28. Zhao Y, Zheng JH, Xu WH, et al. Based on systemic pharmacology and molecular docking to study the mechanism of scallion in the treatment of coronary heart

Cite this article as: Jin Y, Yin X, Li Z, Xu J. Mechanism of Baihe Decoction in the treatment of coronary heart disease based on network pharmacology and molecular docking. Ann Palliat Med 2021;10(3):3205-3218. doi: 10.21037/apm-21-543 disease. Chin J Trad Chin Med 2020;1:105-9.

29. Yang LX, Wang N, Liu Y. Research progress of PI3K/Akt signaling pathway in regulating heart function. J Heart 2018;3:344-7.

30. Ren YC, Zhang L, Jiao XQ. The regulatory effect of sphingosine 1-phosphate on cardiomyocyte apoptosis in coronary heart disease rats through PI3K/Akt signaling pathway. N Chin Med 2020;4:7-10.

31. Pan Y, Yin J, Cai XM, et al. Research progress in the treatment of coronary heart disease with traditional Chinese medicine based on PI3K/Akt signaling pathway. Chin Herb Medi 2017;19:4100-4.

32. Sun J, Nan G. The Mitogen-Activated Protein Kinase (MAPK) Signaling Pathway as a Discovery Target in Stroke. J Mol Neurosci 2016;59:90-8.

33. Zuo C, Zhao X, Shi Y, et al. TNF- $\alpha$ inhibits SATB2 expression and osteoblast differentiation through NF- $\mathrm{NB}$ and MAPK pathways. Oncotarget 2017;9:4833-50.

34. Chen ZX. Association study of MAPK1 and PRKCA gene polymorphisms in MAPK signaling pathway with ischemic stroke and coronary heart disease. Guangxi: Guangxi Medical University, 2019:98-100.

35. Zhang Z, Yao L, Yang J, Wang Z, Du G. PI3K/Akt and HIF 1 signaling pathway in hypoxia ischemia (Review). Mol Med Rep 2018;18:3547-54.

36. Jain T, Nikolopoulou EA, Xu Q, Qu A. Hypoxia inducible factor as a therapeutic target for atherosclerosis. Pharmacol Ther 2018;183:22-33.

(English Language Editor: J. Reynolds) 